\title{
Effect of dexamethasone on complications or all cause mortality after major non-cardiac surgery: multicentre, double blind, randomised controlled trial
}

Check for updates

\begin{abstract}
Karim Asehnoune, ${ }^{1}$ Charlene Le Moal, ${ }^{2}$ Gilles Lebuffe, ${ }^{3}$ Marguerite Le Penndu, ${ }^{1}$ Nolwen Chatel Josse, ${ }^{4}$ Matthieu Boisson, ${ }^{5}$ Thomas Lescot, ${ }^{6}$ Marion Faucher, ${ }^{7}$ Samir Jaber, ${ }^{8}$ Thomas Godet, ${ }^{9}$ Marc Leone, ${ }^{10}$ Cyrus Motamed, ${ }^{11}$ Jean Stephane David, ${ }^{12}$ Raphael Cinotti, ${ }^{13}$ Younes El Amine, ${ }^{14}$ Darius Liutkus, ${ }^{2}$ Matthias Garot, ${ }^{3}$ Antoine Marc, ${ }^{1}$ Anne Le Corre, ${ }^{4}$ Alexandre Thomasseau, ${ }^{5}$ Alexandra Jobert, ${ }^{15}$ Laurent Flet, ${ }^{16}$ Fanny Feuillet, ${ }^{17}$ Morgane Pere, ${ }^{15}$ Emmanuel Futier, ${ }^{9}$ Antoine Roquilly, ${ }^{1}$ on behalf of the PACMAN study group
\end{abstract}

For numbered affiliations see end of the article.

Correspondence to: K Asehnoune karim.asehnoune@chu-nantes.fr (ORCID 0000-0003-1899-3517) Additional material is published online only. To view please visit the journal online.

Cite this as: $B M J$ 2021;373:n1162 http://dx.doi.org/10.1136/bmj.n1162

Accepted: 5 May 2021

\section{ABSTRACT \\ OBJECTIVE}

To assess the effect of dexamethasone on complications or all cause mortality after major noncardiac surgery.

DESIGN

Phase III, randomised, double blind, placebo controlled trial.

\section{SETTING}

34 centres in France, December 2017 to March 2019. PARTICIPANTS

1222 adults ( $>50$ years) requiring major non-cardiac surgery with an expected duration of more than 90 minutes. The anticipated time frame for recruitment was 24 months.

INTERVENTIONS

Participants were randomised to receive either dexamethasone $(0.2 \mathrm{mg} / \mathrm{kg}$ immediately after the surgical procedure, and on day 1) or placebo. Randomisation was stratified on the two prespecified criteria of cancer and thoracic procedure.

MAIN OUTCOMES MEASURES

The primary outcome was a composite of postoperative complications or all cause mortality within 14 days after surgery, assessed in the modified intention-to-treat population (at least one treatment administered).

RESULTS

Of the 1222 participants who underwent randomisation, 1184 (96.9\%) were included in the

\section{WHAT IS ALREADY KNOWN ON THIS TOPIC}

Patients who undergo major surgery are particularly at risk of organ failure from inflammatory origin

In a recent meta-analysis of major abdominal surgery, perioperative use of corticosteroids was associated with a significantly decreased risk of complications

\section{WHAT THIS STUDY ADDS}

Compared with placebo, dexamethasone administered postoperatively at higher dose than the usual antiemetic dose was not associated with a reduction in complications or mortality 14 days after surgery

Use of dexamethasone appeared to be safe

Confidence intervals were, however, wide, and therefore suggests the possibility of important clinical effectiveness modified intention-to-treat population. 14 days after surgery, 101 of 595 participants $(17.0 \%)$ in the dexamethasone group and 117 of 589 (19.9\%) in the placebo group had complications or died (adjusted odds ratio $0.81,95 \%$ confidence interval 0.60 to 1.08; $\mathrm{P}=0.15)$. In the stratum of participants who underwent non-thoracic surgery $(\mathrm{n}=1038)$, the primary outcome occurred in 69 of 520 participants (13.3\%) in the dexamethasone group and 93 of $518(18 \%)$ in the placebo group (adjusted odds ratio $0.70,0.50$ to 0.99). Adverse events were reported in 288 of 613 participants $(47.0 \%)$ in the dexamethasone group and 296 of $609(48.6 \%)$ in the placebo group $(P=0.46)$.

\section{CONCLUSIONS}

Dexamethasone was not found to significantly reduce the incidence of complications and death in patients 14 days after major non-cardiac surgery. The $95 \%$ confidence interval for the main result was, however, wide and suggests the possibility of important clinical effectiveness.

TRIAL REGISTRATION

ClinicalTrials.gov NCT03218553.

\section{Introduction}

More than 300 million major surgical procedures are undertaken each year worldwide. ${ }^{1}$ In a European survey, the mortality rate after non-cardiac surgery was much higher than expected, ${ }^{2}$ and important complications developed in $15 \%$ to $25 \%$ of patients during hospital admission. ${ }^{34}$ Moreover, the impact of postoperative complications on functional status and long term survival is high. As $10 \%$ of patients at risk represent $80 \%$ of postoperative deaths, ${ }^{5}$ approaches targeting high risk patients that even modestly decrease the rate of postoperative complications would considerably improve the long term outcomes of surgical patients and would also lower costs.

Major surgery induces both local and systemic inflammation. ${ }^{6}$ This inflammatory response is a prerequisite for tissue healing, but if it is overwhelmed then remote organ failure or secondary infections can occur. ${ }^{7}$ In this setting, glucocorticoids could be an option, especially given that these molecules have been associated with improved outcomes in medical conditions characterised by systemic inflammatory response, such as septic shock or severe trauma. ${ }^{8-10}$ 
In patients undergoing cardiopulmonary surgery, however, the use of steroids has been associated with an increased risk of myocardial injury, and the effects on atrial fibrillation and postoperative infections remain uncertain. ${ }^{11-13}$

Most studies have investigated the effects on the risk of perioperative nausea of one injection of 4-8 $\mathrm{mg}$ of dexamethasone administered before surgery. ${ }^{14}$ Metaanalyses have concluded that evidence is lacking to show a clear clinical benefit of dexamethasone other than on nausea and vomiting or to rule out major clinical side effects. ${ }^{15} 16$ We hypothesised that a higher dose of dexamethasone could help to safely prevent inflammation related postoperative complications. We conducted the Perioperative Administration of Corticotherapy on Morbidity and mortality After Non-cardiac surgery (PACMAN) phase III randomised controlled trial to assess the effect of dexamethasone on postoperative complications in adults older than 50 years who underwent major non-cardiac surgery.

\section{Methods}

\section{Trial design and setting}

We conducted a pragmatic, investigator initiated, multicentre, parallel group, double blind, randomised controlled trial to compare dexamethasone with placebo in patients considered at risk of complications after major non-cardiac surgery. Patients provided written informed consent before participation. The study protocol and statistical analysis plan were submitted before the inclusion of participants and were published before the end of enrolment. ${ }^{17}$ The department of research at the University hospital of Nantes conducted data monitoring and quality checks. Investigators reported any adverse events within seven days. An independent data and safety monitoring board regularly monitored patient safety and analysed adverse event reports in a blinded manner. All study endpoints were collected and analysed blindly.

The trial was conducted in 34 French hospitals (26 university hospitals, 4 general hospitals, 2 cancer institutes, and 2 private hospitals; see supplementary table A). Patients older than 50 years with at least one risk factor for postoperative complications ${ }^{18}$ or older than 65 years and who were to undergo major noncardiac surgery planned to last 90 minutes or more were eligible for enrolment. The supplementary file provides complete lists of inclusion and exclusion criteria; see study protocol (supplementary table B).

An independent research unit at the University Hospital of Nantes performed the randomisation. A statistician randomised the participants using a computer generated random number in fixed blocks of 6 (1:1 ratio). Stratification was based on surgery for cancer (yes or no) and on intrathoracic surgery (yes or no), both of which are major risk factors for postoperative respiratory complications. ${ }^{18}$ Before surgery was performed, local investigators randomised enrolled participants using a dedicated, password protected, SSL encrypted website (CSOnline; Clinsight) to allow immediate and concealed allocation. At each participating centre, the pharmacist delivered the study drugs, which were then administered by clinical nurses unaware of the treatment groups. Treatment assignment was concealed from participants and site investigators.

\section{Intervention}

The study drug, dexamethasone phosphate $20 \mathrm{mg} / 5$ $\mathrm{mL}$ vials (Mylan; Saint-Priest, France), and placebo were indiscernible. Supplementary table $\mathrm{C}$ describes the blinding process for the experimental drugs.

Participants received dexamethasone $0.2 \mathrm{mg}$ per kilogram of actual body weight (dexamethasone group) or matching placebo (placebo group) as a bolus immediately after surgery ( $<2$ hours after skin closure) and on day 1 after surgery. The maximum daily dose was $20 \mathrm{mg}$.

All other interventions were at the discretion of the clinicians. For consistency, timely antimicrobial prophylaxis, ${ }^{19}$ use of low tidal volume and positive end expiratory pressure during surgery, ${ }^{20}$ treatment of perioperative hypotension, ${ }^{21}$ and early discontinuation of sedation after the procedure ${ }^{22}$ were required. In patients with diabetes, blood glucose levels were measured every 2-3 hours for the first three days, then twice daily for two days, and thereafter the insulin dose was adapted accordingly. Blood levels of troponins in participants considered at risk of postoperative cardiac events were measured according to local procedure. Prophylactic use of glucocorticoids for postoperative nausea and vomiting or postoperative oedema was prohibited. Glucocorticoids were allowed as rescue treatment in case of urgent indications, such as stridor or asthma exacerbation.

\section{Outcomes}

Supplementary table D provides definitions for the outcomes of interest. The primary outcome was a composite of complications and all cause mortality 14 days after surgery. Postoperative complications were sepsis and pneumonia, defined according to consensus criteria, ${ }^{23}$ and the need for invasive or noninvasive mechanical ventilation for acute respiratory failure. ${ }^{2024}$ Each of these outcomes was also analysed separately. Sepsis was defined as life threatening organ dysfunction caused by an infection. ${ }^{23}$ Organ dysfunction was identified as an acute change in total sequential organ failure assessment (SOFA) score of $\geq 2$ points owing to infection. Infection was indicated by an organism identified from blood culture or from a sterile site, or an abscess or infected tissue (eg, pneumonia, peritonitis, urinary tract, vascular line infection, soft tissue).

Secondary outcomes were all cause mortality at 28 days, rates of postoperative complications within 28 days (defined according to the Clavien-Dindo classification), renal and respiratory failures within 14 days after surgery, sequential organ failure assessment score on postoperative days 1 and 3, total duration of invasive and non-invasive mechanical ventilation, lengths of stay in the intensive care unit (ICU) and in 
hospital, and the percentage of patients with adverse events on day 28. C reactive protein concentration was measured after surgery immediately before the first injection of the study treatment, then on days 1 and 2 after surgery.

\section{Analysis}

The rate of the primary outcome ranged from $15 \%$ to $25 \%$ in recent studies. ${ }^{342024}$ In studies including participants at high risk of postoperative complications, dexamethasone was associated with a relative reduction in mortality of $20 \%,{ }^{25}$ respiratory complications of $31 \%$ to $53 \%,{ }^{14}$ and wound infection of $20 \% .{ }^{16}$ We thus hypothesised that dexamethasone would be associated with a relative reduction of $30 \%$ in the rate of our composite primary outcome. Assuming a rate of $20 \%$ in the control group and $14 \%$ in the dexamethasone group, we calculated that a total of 1222 patients (611 patients in each group) would be needed to detect this difference with a 5\% type I error and a power of $80 \%$ in a two sided test.

Analyses were performed using SAS software (version 9.4, NC) before the breaking of the randomisation code. The figures were made with $\mathrm{R}$ version 3.6.1. software. Type I error $(\alpha)$ was set at $5 \%$.

The main analysis of the primary outcome was conducted in the modified intention-to-treat population, ${ }^{17}$ defined as all randomised participants except those who would have no longer been considered eligible for randomisation at the time of first treatment injection or who would never had any injection of the study treatment. We also analysed the primary outcome in the intention-to-treat population, defined as all randomised participants, and finally in the per protocol population, defined as all randomised participants except those with one or more major protocol violations (not eligible for randomisation, received the wrong intervention, surgical intervention was not performed, consent was withdrawn, or received out-of-protocol glucocorticoids). Since data were missing for the primary outcome, we analysed the intention-to-treat population with multiple imputation methods using personal data (age, sex), stratification factors, preoperative biological data, and type of surgery (five imputed datasets).

In the adjusted analyses, we used a logistic regression model that included a fixed effect for stratification factors (cancer, intrathoracic procedure) and centre as a random effect. Kaplan-Meier plots were used to show the rate of events in a time-to-event analysis, and differences were tested using a Cox model adjusted on stratification factors and centre as random effect and censored at 14 days after surgery. Independent components of the primary outcome (other than death) were analysed with Fine and Gray models adjusted on stratification factors, and centre as random effect with death considered as a competing risk. For analysis of the prespecified subgroups, we calculated the odds ratios with $95 \%$ confidence intervals without multiple adjustment. The direction effect across the subgroups was not a priori indicated in the protocol.
Analyses of secondary outcomes were conducted on data from the modified intention-to-treat population and took into account stratified randomisation and centre as random effect. Continuous variables are presented as means and standard deviations or as medians and interquartile ranges, and categorical data are presented as numbers and percentages. Missing data are described by treatment arm.

Categorical data (eg, the proportion of patients who experienced adverse events, postoperative morbidity, primary outcome) wereanalysed with logistic regression adjusted for stratification factors as fixed effect and centre as a random effect. Ordinal categorical data (infection severity and Clavien-Dindo classification) were analysed with ordinal logistic regression adjusted for stratification factors. Longitudinal continuous data were analysed with linear mixed models, with random effects models adjusted for stratification factors to account for repeated measurements. Assumptions of normality and homoscedasticity associated with these models were evaluated. The duration of mechanical ventilation and stays in the ICU and hospital were analysed using competing risk models to take into account informative censoring and competing risk owing to death.

\section{Patient and public involvement}

Except for providing written informed consent before participation, no patients or members of the public were involved in the research, mainly because of funding restrictions. Although patients and the public were not directly involved in the study, we did speak to patients about the study and we asked a member of the public to read our manuscript before submission.

\section{Results}

From December 2017 to March 2019, 1222 participants were randomised (613 in the dexamethasone group and 609 in the placebo group; fig 1). No unblinding occurred, and 13 of 1222 participants (1.1\%) received dexamethasone outside of the study protocol. After excluding 38 participants (did not meet the inclusion criteria, did not receive any injection of the experimental treatment, or withdrew consent), 595 participants in the dexamethasone group and 589 in the placebo group met the criteria for the modified intention-to-treat population. Thirty eight of 1222 participants $(3.1 \%)$ had major protocol violations (received the wrong intervention, no surgical intervention was performed, or withdrew consent) but were kept in the modified intention-to-treat analysis (see supplementary table E for protocol dropouts). Table 1 shows the baseline characteristics of the participants. Most types of surgery were represented. Clinical care outside the trial intervention, including antimicrobial prophylaxis, haemodynamic support, and perioperative ventilatory support, complied with the international standard of care recommendations.

In the modified intention-to-treat analysis, 1184 of 1222 participants (96.9\%) were analysed. Overall, 101 of 595 participants $(17.0 \%)$ in the dexamethasone 


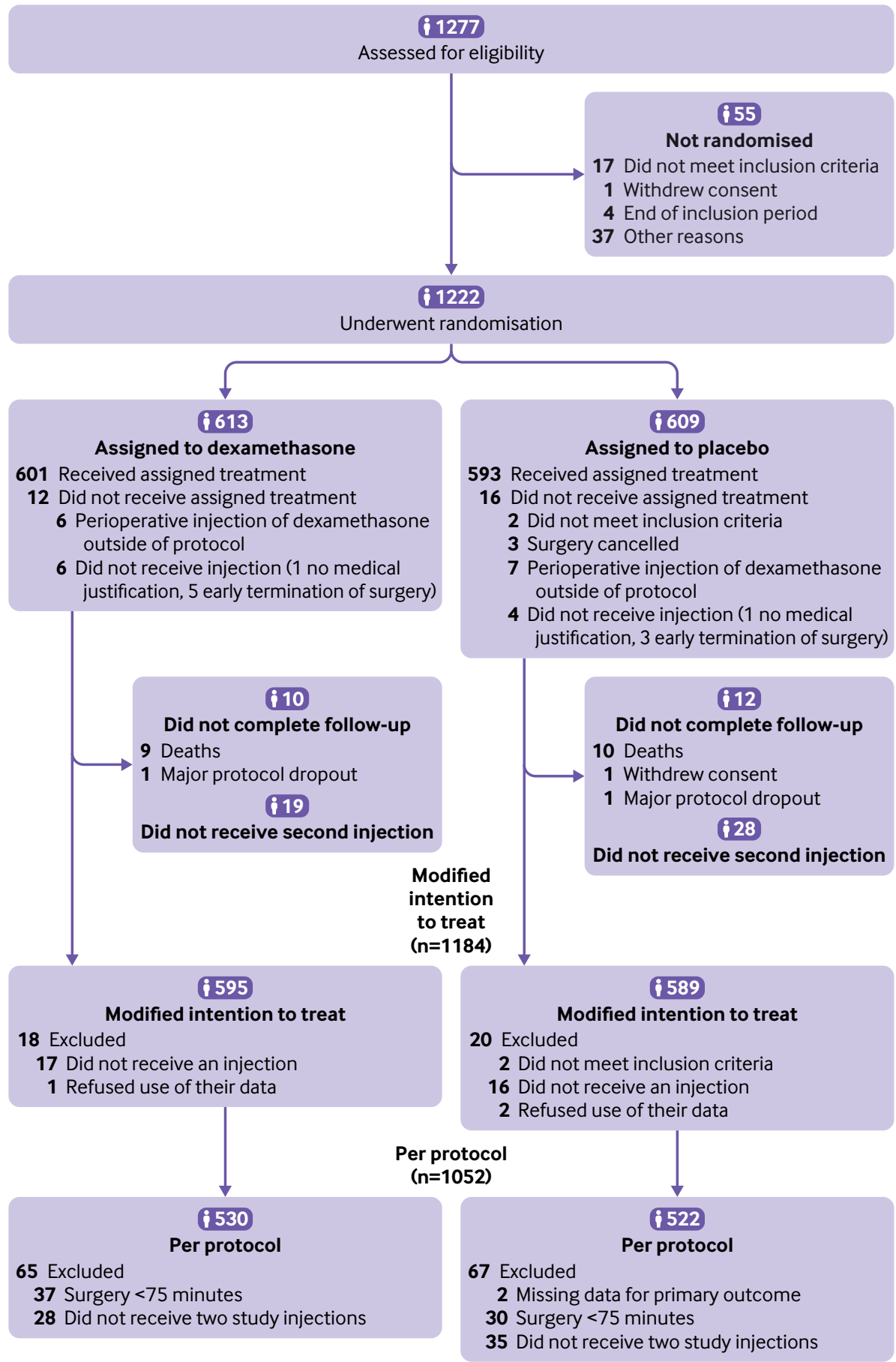

Fig 1 | Flow of participants through study

group and 117 of 589 (19.9\%) in the placebo group had died or developed complications 14 days after surgery (adjusted odds ratio $0.81,95 \%$ confidence interval 0.60 to 1.08 ; $\mathrm{P}=0.15$, table 2 ). The adjusted hazard ratio for the composite of postoperative complications and all cause mortality with dexamethasone was 0.74 ( 0.64 to $1.09 ; \mathrm{P}=0.18$, see supplementary fig A). Supplementary figure B shows the range of sample sizes across centres, and the centre specific estimates of odd ratios for the primary outcome with dexamethasone.
In the a priori defined randomisation stratum of participants who underwent non-thoracic surgery $(n=1038)$, the composite of postoperative complications and all cause mortality occurred in 69 of 520 participants $(13.3 \%)$ in the dexamethasone group and 93 of $518(18.0 \%)$ in the placebo group (adjusted odds ratio $0.70,0.50$ to 0.99 , fig 2 ). In the other a priori stratum of randomisation, dexamethasone was not associated with a change in risk of the primary outcome in participants who did or did not have surgery for cancer $(0.79,0.56$ to 1.11 and $0.95,0.47$ to 


\begin{tabular}{|c|c|c|c|}
\hline Characteristics & Total $(n=1184)$ & Dexamethasone group $(n=595)$ & Placebo group $(n=589)$ \\
\hline Median (interquartile range) age (years) & $69(65-74)$ & $69(65-74)$ & $70(65-74)$ \\
\hline Men & $748(63.2)$ & $377(63.4)$ & $371(63)$ \\
\hline Median (interquartile range) body mass index & $26.2(23.1-29.4)$ & $26.1(23.1-29.6)$ & $26.3(23.1-29.4)$ \\
\hline Medical history: & & $\mathrm{n}=560$ & $n=561$ \\
\hline Arterial hypertension & $645(57.5)$ & $325(58)$ & $320(57)$ \\
\hline Diabetes mellitus & $233(20.8)$ & $120(21.4)$ & $113(20.1)$ \\
\hline Cardiac insufficiency & $67(6)$ & $36(6.4)$ & $31(5.5)$ \\
\hline Coronary heart disease & $129(11.5)$ & $66(11.8)$ & $63(11.2)$ \\
\hline Chronic pulmonary disease & $143(12.8)$ & $73(13)$ & $70(12.5)$ \\
\hline Chronic renal failure & $81(7.2)$ & $37(6.6)$ & $44(7.8)$ \\
\hline Stroke & $74(6.6)$ & $36(6.4)$ & $38(6.7)$ \\
\hline Current or former smoker & $146(13)$ & $83(14.8)$ & $63(11.2)$ \\
\hline Malnutrition & $89(7.9)$ & $40(7.1)$ & $49(8.7)$ \\
\hline Preoperative factors: & & $n=595$ & \\
\hline Nutritional support & $230(19.4)$ & $106(17.8)$ & $124(19.4)$ \\
\hline Chemotherapy & $206(17.4)$ & $111(18.7)$ & $95(16.1)$ \\
\hline American Society of Anesthesiology score: & & $\mathrm{n}=595$ & \\
\hline 1 & $80(6.8)$ & $33(5.6)$ & $47(8)$ \\
\hline II & $713(60.2)$ & $367(61.7)$ & $346(58.7)$ \\
\hline III & $380(32.1)$ & $190(31.9)$ & $190(32.2)$ \\
\hline IV & $11(0.9)$ & $5(0.8)$ & $6(1)$ \\
\hline Median (interquartile range) blood test result at inclusion: & & $n=525$ & $n=528$ \\
\hline Leucocytes $\left(\times 10^{9} / \mathrm{L}\right)$ & $7.9(5.6-8.5)$ & $7.9(5.6-8.6)$ & $7.4(5.6-8.3)$ \\
\hline Neutrophils $\left(\times 10^{9} / \mathrm{L}\right)$ & $4.3(3.2-5.5)$ & $4.3(3.2-5.7)$ & $4.3(5.2-5.3)$ \\
\hline Lymphocytes $\left(\times 10^{9} / \mathrm{L}\right)$ & $1.7(1.3-2.2)$ & $1.7(1.2-2.3)$ & $1.7(1.3-2.1)$ \\
\hline Creatinine $(\mu \mathrm{mol} / \mathrm{L})$ & $73(62-88)$ & $73(62-88)$ & $73(62-88)$ \\
\hline Incision type: & & $n=595$ & $\mathrm{n}=589$ \\
\hline Laparoscopy & $381(32.2)$ & $196(32.9)$ & $185(31.4)$ \\
\hline Laparotomy & $889(75.1)$ & $440(74)$ & $449(76.2)$ \\
\hline Surgery type: & & $n=595$ & \\
\hline Cancer & $774(65.4)$ & $385(64.7)$ & $389(66)$ \\
\hline Intrathoracic & $146(12.3)$ & $75(12.6)$ & $71(12.1)$ \\
\hline Abdominal & $752(63.5)$ & $385(64.7)$ & $367(62.3)$ \\
\hline Vascular & $68(5.7)$ & $30(5)$ & $38(6.5)$ \\
\hline Orthopaedic & $174(14.7)$ & $92(15.5)$ & $82(13.9)$ \\
\hline Neck or face & $81(6.8)$ & $47(7.9)$ & $34(5.8)$ \\
\hline Antimicrobial prophylaxis & $1056(89.2)$ & $541(90.9)$ & $515(87.4)$ \\
\hline No antimicrobial prophylaxis by surgery type: & & $\mathrm{n}=595$ & \\
\hline Cancer & $96(8.9)$ & $39(6.6)$ & $57(9.7)$ \\
\hline Intrathoracic & $5(0.5)$ & $1(0.2)$ & $4(0.7)$ \\
\hline Abdominal & $61(5.6)$ & $28(4.7)$ & $33(5.6)$ \\
\hline Vascular & $4(0.4)$ & $2(0.3)$ & $2(0.3)$ \\
\hline Orthopaedic & $1(0.1)$ & $0(0.0)$ & $1(0.2)$ \\
\hline Neck or face & $15(1.4)$ & $9(1.5)$ & $6(1.0)$ \\
\hline Warming blanket & $1119(94.5)$ & $557(93.6)$ & $562(95.4)$ \\
\hline \multicolumn{4}{|l|}{ Mechanical ventilation during procedure: } \\
\hline Median (interquartile range) tidal volume $(\mathrm{mL})$ & $470(425-510)$ & $470(430-510)$ & $470(420-510)$ \\
\hline Median (interquartile range) PEEP $\left(\mathrm{cmH}_{2} \mathrm{O}\right)$ & $5(4-6)$ & $5(4-6)$ & $5(5-6)$ \\
\hline Recruitment manoeuvre & $593(50.1)$ & $295(49.7)$ & $298(50.6)$ \\
\hline Haemodynamic support: & & $\mathrm{n}=595$ & \\
\hline Stroke volume monitoring & $203(17.1)$ & $98(16.5)$ & $105(17.8)$ \\
\hline Blood transfusion & $110(9.3)$ & $50(8.4)$ & $60(10.2)$ \\
\hline Locoregional analgesia: & & $n=525$ & $n=534$ \\
\hline Spinal & $63(11.2)$ & $35(12)$ & $28(10.3)$ \\
\hline Peridural & $236(41.8)$ & $117(40.2)$ & $119(43.6)$ \\
\hline Perineural & $121(21.5)$ & $58(19.9)$ & $63(23.1)$ \\
\hline Scar infiltration & $150(26.6)$ & $85(29.2)$ & $65(23.8)$ \\
\hline Intraoperative complications: & & $n=595$ & $\mathrm{n}=589$ \\
\hline Allergic reaction & $85(7.2)$ & $41(6.9)$ & $44(7.5)$ \\
\hline Haemorrhagic shock & $30(2.5)$ & $14(2.3)$ & $16(2.7)$ \\
\hline Organ perforation & $7(0.6)$ & $3(0.5)$ & $4(0.7)$ \\
\hline Median (interquartile range) duration of surgery (mins) & $188(119-296)$ & $195(120-305)$ & $180(115-288)$ \\
\hline $\begin{array}{l}\text { Median (interquartile range) delay between skin } \\
\text { closure and study treatment injection (mins) }\end{array}$ & $10(0-29)$ & $10(0-0.30)$ & $10(0-0.30)$ \\
\hline $\begin{array}{l}\text { Median (interquartile range) dose of } \\
\text { dexamethasone or placebo }(\mathrm{mg})\end{array}$ & $15(13-17)$ & $15(13-17)$ & $15(13-17)$ \\
\hline
\end{tabular}

PEEP=positive end expiratory pressure. 
1.88, respectively; fig 2). The effect of dexamethasone on risk of the primary outcome did not appear to be modified in any of the other prespecified subgroups, including duration of surgery, $\mathrm{C}$ reactive protein level on day 0 , age, history of diabetes, site of incision, and surgery type (fig 2).

In the intention-to-treat analysis including all randomised participants, little change was found in the primary outcome $(0.77,0.57$ to 1.05$)$. Supplementary table $\mathrm{F}$ presents the analysis of participants with missing data. In the per protocol population, the adjusted odds ratio of the primary outcome with dexamethasone was 0.84 (0.61 to 1.16).

The mortality rate on day 14 was $1.0 \%$ (6 of 595 participants) in the dexamethasone group and 1.2\% (7 of 589) in the placebo group; adjusted odds ratio with dexamethasone was 0.84 ( 0.52 to 1.38 ; table 2$)$. The adjusted odds ratio for sepsis or pneumonia, or both, with dexamethasone was 0.82 (0.60 to 1.11) and the need for mechanical ventilation was 0.70 (0.53 to 0.93; table 2).

In the ordinal analyses for rates of complications using the Clavien-Dindo classification, no differences were found between the two study groups $(0.94,0.75$ to 1.17; table 2). Dexamethasone was associated with reduced rates of postoperative vomiting and acute kidney injury (0.66, 0.44 to 0.99 and $0.52,0.30$ to 0.91 , respectively; table 2 ). Postoperative $C$ reactive protein blood levels showed a significant decrease in the dexamethasone group compared with placebo group (adjusted estimate -3.37 , 95\% confidence interval -5.65 to -1.09$)$. No statistically significant differences were found for the other secondary outcomes (table 2).

Safety was assessed in the intention-to-treat population (table 3 , also see supplementary table G for a complete list of severe adverse events). Nine of 613 participants $(1.5 \%)$ in the dexamethasone group and 12 of $609(2.0 \%)$ in the placebo group received glucocorticoids as rescue treatment. The numbers of participants experiencing adverse events were 288 of $613(47.0 \%)$ in the dexamethasone group and 296 of $609(48.6 \%)$ in the placebo group (odds ratio 0.92, $95 \%$ confidence interval 0.74 to $1.15, \mathrm{P}=0.46)$. The rates of normal healing, delayed healing, and surgical wound dehiscence did not differ between the two groups $(\mathrm{P}=0.79, \mathrm{P}=0.50$, and $\mathrm{P}=0.58$, respectively). In the dexamethasone group, 166 of 613 participants (27.4\%) required insulin for hyperglycaemia compared with 131 patients of $609(21.5 \%)$ in the placebo group (odds ratio 1.36, 0.99 to $1.88 ; \mathrm{P}=0.06$ ).

\section{Discussion}

In this multicentre, double blind, randomised controlled trial of patients undergoing major non-cardiac surgery, dexamethasone administered postoperatively was not associated with a significant reduction in complications and mortality at 14 days after surgery.

Dexamethasone was, however, associated with a significant reduction in the rate of complications and all cause mortality at 14 days after surgery in the a priori stratum of participants who did not undergo an intrathoracic procedure. In this population, respiratory complications were probably mainly caused by excessive systemic postoperative inflammation, whereas lung complications after intrathoracic procedures probably relate to atelectasis caused by direct tissue damage and are therefore probably less responsive to systemic corticosteroids. The result of the subgroup analysis suggests caution in using dexamethasone in patients after intrathoracic surgery.

Two mechanisms might explain why corticosteroids could decrease the risk of major complications after surgery. Firstly, the potent anti-inflammatory effects of dexamethasone might reduce the excessive postoperative inflammatory response that results in remote organ failure and immune cell apoptosis. In support of this mechanism, we observed a major decrease in $\mathrm{C}$ reactive protein blood concentrations in the dexamethasone group compared with placebo group. We decided to administer dexamethasone postoperatively at a time when inflammation had already been initiated, because exogeneous corticosteroids are associated with leucocyte apoptosis during homoeostasis but are immunostimulant during inflammation. ${ }^{26}$ Secondly, glucocorticoids might also restore immune functions, which are decreased in patients admitted to hospital at risk of secondary infections. ${ }^{27}$ When glucocorticoids are administered during a systemic inflammatory response, blood concentrations of anti-inflammatory cytokines decrease, concentrations of interferon $\gamma$ and interleukin-12 increase, and the phagocytic abilities of neutrophils improves. ${ }^{28-30}$ The use of moderate doses of corticosteroids to reduce the risk of infection is probably counterintuitive. In our study, however, the relative risk for the primary outcome measure in patients treated with dexamethasone was 0.81 , which is within the $95 \%$ confidence intervals of the estimated effects of glucocorticoids for the treatment or prevention of sepsis. ${ }^{10} 31$ Notably, corticosteroids have been shown to reduce the risk of early death in patients with septic shock. ${ }^{31}$ In acute inflammatory processes, corticosteroids have been shown to reduce the risk of pneumonia after severe trauma ${ }^{9}$ and after traumatic brain injury, ${ }^{10}$ and dexamethasone has been shown to decrease the severity of community acquired pneumonia. ${ }^{32}{ }^{33}$ Respiratory problems are among the most common complications after major surgery, ${ }^{4} 20$ and the present results suggest that dexamethasone could help to reduce the need for mechanical ventilation for respiratory failure postoperatively.

\section{Comparison with other studies}

Results from a meta-analysis of the perioperative use of corticosteroids after major abdominal surgery ${ }^{34}$ comprising 439 patients from 11 randomised controlled trials suggested that corticosteroids could decrease the risk of major complications. In another meta-analysis, comprising 381 patients, however, corticosteroids were not associated with a reduced risk of pulmonary complications after transthoracic 


\begin{tabular}{|c|c|c|c|c|}
\hline Outcomes & $\begin{array}{l}\text { Dexamethasone group } \\
(\mathrm{n}=595)\end{array}$ & $\begin{array}{l}\text { Placebo group } \\
(n=589)\end{array}$ & Estimate $(95 \% \mathrm{Cl})$ & $P$ value \\
\hline Primary outcome: complications and mortality at 14 days & $101(17.0)$ & $117(19.9)$ & $0.81(0.60 \text { to } 1.08)^{\star}$ & 0.15 \\
\hline All cause mortality & $6(1.0)$ & $7(1.2)$ & $0.84(0.52$ to 1.38$) \dagger$ & 0.5 \\
\hline Postoperative pneumonia or sepsis, or both & $78(13.1)$ & $94(16.0)$ & $0.82(0.60$ to 1.11$) \ddagger$ & 0.2 \\
\hline Mechanical ventilation for respiratory failure & $41(6.9)$ & $52(8.8)$ & $0.70(0.53$ to 0.93$) \neq$ & 0.015 \\
\hline \multicolumn{5}{|l|}{ Infection localisation: } \\
\hline Pneumonia & $13(2.1)$ & $18(3.1)$ & $0.63(0.31 \text { to } 1.31)^{\star}$ & \\
\hline Surgical site & $52(8.7)$ & $57(9.7)$ & $1.13(0.63 \text { to } 2.02)^{*}$ & \\
\hline Septicaemia & $6(1.0)$ & $11(1.9)$ & $0.65(0.23 \text { to } 1.85)^{*}$ & \\
\hline Urinary tract & $14(2.4)$ & $14(2.4)$ & $1.32(0.64 \text { to } 2.75)^{*}$ & \\
\hline Infection severity: & & & $0.85(0.45$ to 1.61$) \S$ & \\
\hline Sepsis & $67(74.4)$ & $75(72.8)$ & & \\
\hline Severe sepsis & $17(18.9)$ & $18(17.5)$ & & \\
\hline Septic shock & $6(6.7)$ & $10(9.7)$ & & \\
\hline \multicolumn{5}{|l|}{ Non-invasive mechanical ventilation: } \\
\hline Day 14: & $33(5.6)$ & $46(7.8)$ & $0.67(0.41 \text { to } 1.09)^{\star}$ & \\
\hline Median (interquartile range) duration (days) & $3(2-5)$ & $6(2-9)$ & $0.69(0.51$ to 0.94$) \neq$ & \\
\hline \multicolumn{5}{|l|}{ Invasive mechanical ventilation: } \\
\hline Day 14 & $15(2.5)$ & $18(3.1)$ & $0.80(0.40 \text { to } 1.64)^{\star}$ & \\
\hline Median (interquartile range) duration of mechanical ventilation (days) & $2(0-7)$ & $2(1-4)$ & 0.76 (0.43 to 1.32$) \ddagger$ & \\
\hline Clavien-Dindo grade at day 28: & & & $0.94(0.75$ to 1.17$) \S$ & \\
\hline 0 (no complications) & $340(57.1)$ & $315(53.6)$ & & \\
\hline 1 (no intervention) & $34(5.7)$ & $37(6.3)$ & & \\
\hline 2 (drug intervention) & $117(19.7)$ & $141(24)$ & & \\
\hline 3a-b (radio-intervention or surgery) & $74(12.4)$ & $63(10.7)$ & & \\
\hline 4a-b (ICU admission) & $19(3.2)$ & $21(3.6)$ & & \\
\hline 5 (death) & $11(1.9)$ & $11(1.9)$ & & \\
\hline Mean (SD) SOFA score: & $\mathrm{n}=546$ & $\mathrm{n}=541$ & $0.02(-0.12$ to 0.17$)$ q & \\
\hline \multirow[t]{2}{*}{ Day 1} & $0.6(1.4)$ & $0.6(1.4)$ & & \\
\hline & $\mathrm{n}=511$ & $n=511$ & & \\
\hline \multirow[t]{2}{*}{ Day 3} & $0.5(1.3)$ & $0.5(1.3)$ & & \\
\hline & $n=595$ & $n=589$ & & \\
\hline Acute respiratory distress syndrome & $11(1.9)$ & $14(2.4)$ & $0.78(0.42 \text { to } 1.45)^{\star}$ & \\
\hline Postoperative acute kidney injury (KDIGO $\geq 2)$ & $17(2.9)$ & $33(5.6)$ & $0.52(0.30 \text { to } 0.91)^{\star}$ & \\
\hline Median (interquartile range) blood C reactive protein $(\mathrm{mg} / \mathrm{mL}$ ): & & & $-3.37(-5.65$ to -1.09$) 9$ & \\
\hline Day 0 (before 1st injection) & $4(2$ to 7$)$ & $4(1$ to 7$)$ & & \\
\hline Day 1 & $54(26-98)$ & $82(46-129)$ & & \\
\hline Day 2 & $53(25-97)$ & $133(85-206)$ & & \\
\hline Vomiting & $51(8.6)$ & $73(12.4)$ & $0.66(0.44 \text { to } 0.99)^{*}$ & \\
\hline \multicolumn{5}{|l|}{ ICU admission: } \\
\hline Total & $298(50.1)$ & $290(49.3)$ & $1.06(0.82 \text { to } 1.36)^{\star}$ & \\
\hline Scheduled & $276(93.2)$ & $265(92.3)$ & $1.11(0.59 \text { to } 2.10)^{\star}$ & \\
\hline Emergency & $31(10.5)$ & $27(9.4)$ & $1.12(0.64 \text { to } 1.95)^{\star}$ & \\
\hline Unplanned or readmission & $36(12.1)$ & $34(11.7)$ & $1.05(0.64 \text { to } 1.74)^{\star}$ & \\
\hline Median (interquartile range) duration of ICU stay (days) & $0(0-4)$ & $0(0-5)$ & $1.03(0.89$ to 1.19$) \ddagger$ & \\
\hline Median (interquartile range) duration of hospital stay (days) & $27(20-28)$ & $27(21-28)$ & $1.03(0.92$ to 1.14$) \ddagger$ & \\
\hline \multicolumn{5}{|l|}{ Postoperative morbidity at day 7 : } \\
\hline Acute kidney injury (KDIGO 2-3) & $4(0.7)$ & $10(1.7)$ & $0.39(0.12 \text { to } 1.23)^{\star}$ & \\
\hline Acute coronary syndrome & $1(0.2)$ & $0(0.0)$ & 1 & \\
\hline Infection & $58(9.8)$ & $68(11.5)$ & $0.83(0.56 \text { to } 1.23)^{\star}$ & \\
\hline Pneumonia & $11(1.9)$ & $16(2.7)$ & $0.66(0.31 \text { to } 1.44)^{\star}$ & \\
\hline Surgical site infection & $34(5.7)$ & $34(5.8)$ & $0.99(0.59 \text { to } 1.67)^{\star}$ & \\
\hline Sepsis & $58(5.8)$ & $68(11.5)$ & $0.83(0.56 \text { to } 1.23)^{\star}$ & \\
\hline Septic shock & $2(0.3)$ & $6(1.0)$ & & \\
\hline \multicolumn{5}{|c|}{$\begin{array}{l}\text { ICU=intensive care unit; SOFA=sepsis related organ failure assessment score; KDIGO=Kidney Disease: Improving Global Outcomes. } \\
\text { *Marginal odds ratio calculated with logistic regression model adjusted on stratification factors and centres as random effect. } \\
\text { †Marginal hazard ratio calculated with cox model adjusted on stratification factors and centres as random effect. } \\
\text { ¥Marginal hazard ratio calculated with competitive risk survival model adjusted on stratification factors and centres as random effect. } \\
\text { §Marginal odds ratio calculated with ordinal logistic regression model adjusted on stratification factors and centres as random effect. } \\
\text { १Marginal estimate calculated with mixed linear regression adjusted on stratification factors and centres as random effect. }\end{array}$} \\
\hline
\end{tabular}

oesophagectomy. ${ }^{35}$ In both meta-analyses, ${ }^{34}{ }^{35}$ use of corticosteroids was not associated with adverse effects compared with placebo, and the authors suggested the need for larger population samples in future randomised controlled trials.
Concerns about the potential side effects of dexamethasone have emerged-notably, the risk of postoperative anastomotic leakage, delayed healing, and metabolic disorders such as hyperglycaemia. ${ }^{16} 25$ These concerns are important because $30 \%$ to 


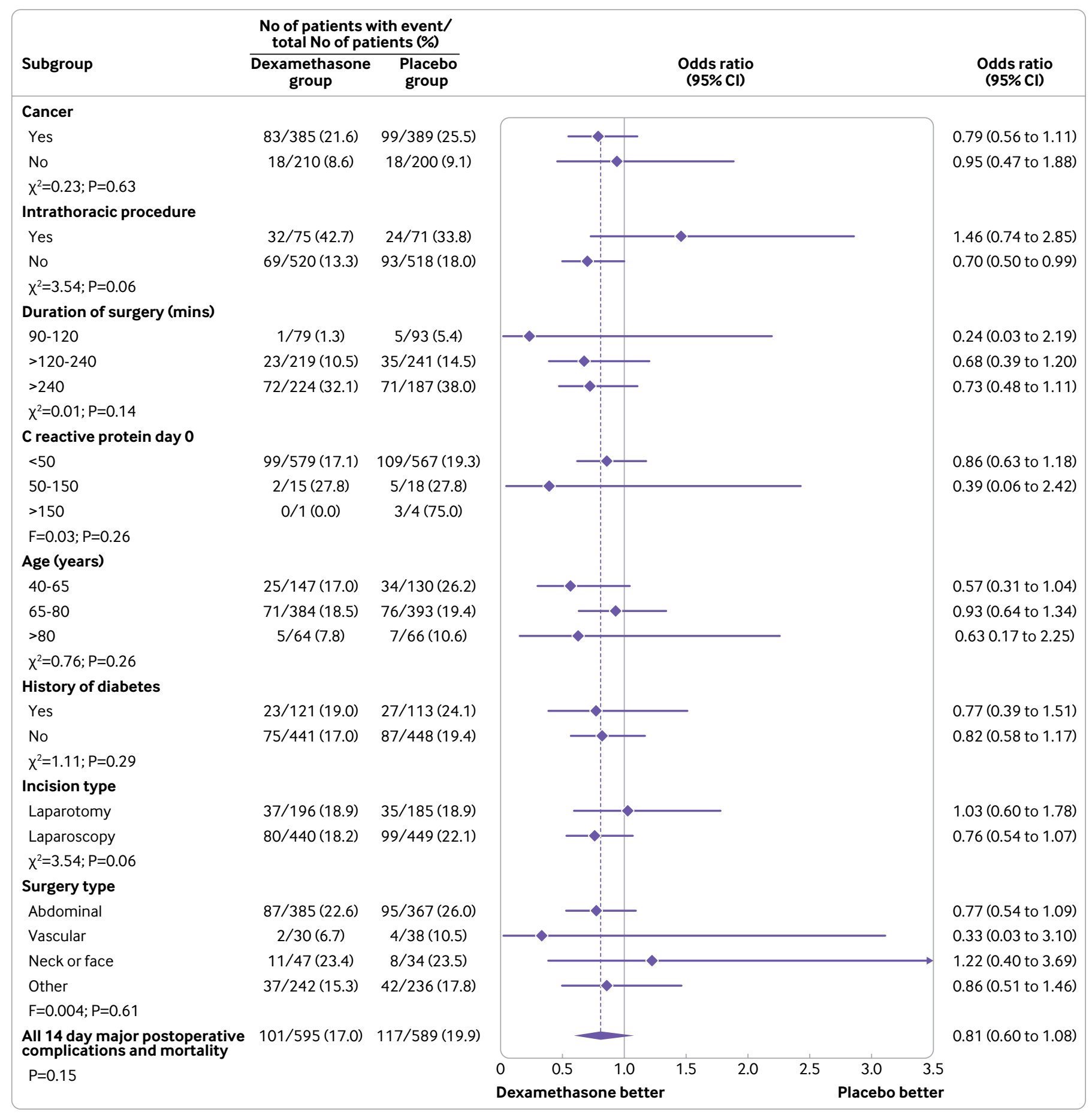

Fig 2 | Subgroup analysis of complications or all cause mortality at 14 days after major non-cardiac surgery. Size of square reflects the relative numbers in each subgroup, and horizontal bars are $95 \%$ confidence intervals. It was not possible to analyse the subgroup $C$ reactive protein $>150 \mathrm{mg} / \mathrm{mL}$

$80 \%$ of patients undergoing surgery will receive dexamethasone perioperatively for antiemetic prophylaxis. ${ }^{36}$ With the exception of hyperglycaemia and transient requirement for insulin, our results show that even at higher doses than the classic antiemetic regimen used in surgery, dexamethasone is well tolerated postoperatively-confirming the results obtained by the Cochrane review group. ${ }^{15}$ It has been suggested that perioperative hyperglycaemia is associated with adverse outcomes in general surgery patients with and without diabetes. The risk of an adverse event, however, has been linked to hyperglycaemia only in patients without diabetes, and this is probably related to the underuse of insulin in these patients. ${ }^{37-39}$ The clinical relevance of the mild and transient hyperglycaemia reported after dexamethasone use is questionable. We observed that a reasonable increase in the dose of insulin within the 


\begin{tabular}{|c|c|c|c|c|}
\hline & $\begin{array}{l}\text { Dexamethasone } \\
\text { group }(n=613)\end{array}$ & $\begin{array}{l}\text { Placebo group } \\
(n=609)\end{array}$ & Estimate $(95 \% \mathrm{Cl})$ & $P$ values \\
\hline Adverse events & $288(47.0)$ & $296(48.6)$ & $0.92(0.74 \text { to } 1.15)^{\star}$ & 0.46 \\
\hline Severe adverse events & $106(17.3)$ & $103(16.9)$ & $1.03(0.75 \text { to } 1.42)^{\star}$ & 0.86 \\
\hline \multicolumn{5}{|l|}{ Gastrointestinal adverse events: } \\
\hline Anastomotic leakage & $17(2.8)$ & $23(3.8)$ & $0.70(0.38 \text { to } 1.31)^{\star}$ & 0.27 \\
\hline \multicolumn{5}{|l|}{ Metabolic disorderst: } \\
\hline Hypokalaemia & $74(12.4)$ & $110(18.8)$ & $0.59(0.43 \text { to } 0.81)^{\star}$ & 0.001 \\
\hline Hyponatraemia & $164(27.4)$ & $144(24.6)$ & $1.14(0.89 \text { to } 1.45)^{\star}$ & 0.31 \\
\hline Hypernatraemia & $8(1.3)$ & $20(3.4)$ & $0.38(0.17 \text { to } 0.86)^{\star}$ & 0.02 \\
\hline Day 2 & $7.7(6.4-9.2)$ & $7.0(6.0-8.2)$ & & \\
\hline Day 3 & $6.2(5.2-7.6)$ & $6.4(5.5-7.8)$ & & \\
\hline Insulin treatment & $166(27.4)$ & $131(21.5)$ & $1.36(0.99 \text { to } 1.88)^{\star}$ & 0.06 \\
\hline $\begin{array}{l}\text { Median (interquartile range) } \\
\text { total dose of insulin (IU/day): }\end{array}$ & & & $-0.97(-5.73$ to 3.78$) \ddagger$ & 0.69 \\
\hline Day 1 & $27.0(12.0-46.0)$ & $24.0(11.0-44.0)$ & & \\
\hline Day 2 & $24.0(12.0-42.0)$ & $24.0(9.0-38.0)$ & & \\
\hline Day 3 & $16.0(3.0-30.5)$ & $15.5(4.0-37.5)$ & & \\
\hline \multicolumn{5}{|l|}{ Healing: } \\
\hline Normal & $541(89.6)$ & $536(89.9)$ & $1.05(0.72 \text { to } 1.54)^{\star}$ & 0.79 \\
\hline
\end{tabular}

first 48 hours enabled normalisation of the glycaemia. We did not find an association between a history of diabetes and the effect of dexamethasone on the primary outcome. In accordance with our data, it has been shown recently that high doses of dexamethasone administered perioperatively to children after cardiac surgery was not associated with major harm. ${ }^{40}$

\section{Strengths and limitations of this study}

The strengths of this double blind, randomised controlled trial include its large size and the number of participating sites. To reduce variability in the perioperative care of patients, the clinicians were asked to follow local recommendations for antimicrobial prophylaxis, protective ventilation (low tidal volume ventilation), prevention of hypotension, and duration of sedation. We evaluated a wide variety of surgical interventions and therefore our results should be considered as highly representative of daily practice. Our study did, however, have some limitations. Firstly, the study dose of dexamethasone was higher than that recommended for the prevention of nausea and vomiting. Interestingly, the range of dexamethasone in equivalent hydrocortisone was $200-400 \mathrm{mg} /$ day, which is close to the dose administered in the ICU for septic shock or trauma. ${ }^{41}$ Our results do, however, suggest that dexamethasone should be used with caution in patients undergoing intrathoracic procedures; a further limitation is the lack of patient and public involvement. Secondly, we used a composite primary outcome, which cannot show a reduction in each specific postoperative complication; our score has not been previously validated and did not include postoperative cardiac complications. Also, the primary outcome was evaluated within 14 days of surgery and a slightly longer period could have been chosen. Thirdly, the modified intention-to-treat analysis, which only included participants who had received at least one dose of treatment, was chosen as the primary analysis because we aimed to evaluate for the first time a new dose of dexamethasone administered postoperatively to treat complications related to the inflammatory response; however, as inflammation commences at the time of tissue injury from skin incision, an invaluable approach might have been to administer dexamethasone earlier. Fourthly, participants were randomised in a fixed block size, whereas the use of random permuted block size could have helped to reduce the risk of selection bias. Moreover, the randomisation was not stratified on centres, which could theoretically affect the overall balance of the treatment groups. Yet, the treatment effects varied little across the centres. Finally, the study power was perhaps too small to show an effect of dexamethasone on the primary outcome. We aimed to reduce postoperative inflammation using dexamethasone and hypothesised that the treatment effect would be consistent across a wide range of prolonged surgery. The subgroup analyses, however, suggested that the heterogeneity of the surgery and patients had probably contributed to an imprecise estimation of the treatment effect, reducing the study power. The effect size used for the power calculation was based on high risk patients but could have been overestimated. With 
a $19.9 \%$ rate in the control group and $17.0 \%$ in the dexamethasone group, a total of 5616 patients would have been needed to detect this difference between the two groups with a 5\% type I error and a power of $80 \%$ in a two sided test.

\section{Conclusion}

Postoperative treatment with dexamethasone was not associated with a significant reduction in the incidence of complications and death in patients undergoing major non-cardiac surgery. The 95\% confidence interval for the main result was, however, wide and therefore suggests the possibility of important clinical effectiveness. At present, the use of glucocorticoids to prevent postoperative systemic complications is not widely adopted. Our trial described an innovative regimen adapted to a patient's body weight that was well tolerated postoperatively.

\section{AUTHOR AFFILIATIONS}

${ }^{1} \mathrm{CHU}$ Nantes, Université de Nantes, Pôle Anesthésie-Réanimation, Service d'Anesthésie Réanimation Chirurgicale, Hôtel Dieu, Nantes, France

${ }^{2}$ Service d'Anesthésie, Centre Hospitalier Le Mans, Le Mans, France ${ }^{3}$ Centre Hospitalier Universitaire (CHU) Lille, Pôle Anesthésie Réanimation, Lille, France

${ }^{4}$ Service d’Anesthésie, Hôpital Privé du Confluent, Nantes, France ${ }^{5} \mathrm{CHU}$ de Poitiers, Université de Poitiers, Service d’AnesthésieRéanimation, Poitiers, France

${ }^{6}$ Hôpital Saint Antoine, Service d'Anesthésie Réanimation Chirurgicale, Assistance publique des hôpitaux de Paris, Paris, France

${ }^{7}$ Institut Paoli Calmette, Service d'Anesthésie, Marseille, France

${ }^{8}$ Anesthesia and Critical Care Department B, Saint Eloi Teaching Hospital, PhyMedExp, Centre Hospitalier Universitaire Montpellier, University of Montpellier, INSERM U1046, CNRS UMR 9214. Montpellier, France

${ }^{9}$ Service d'Anesthésie et Réanimation, Hôpital Estaing, CHU Clermont Ferrand, Clermont-Ferrand, France

${ }^{10}$ Department of Anesthesiology and Critical Care Medicine, Hôpital Nord, Aix Marseille University, Assistance Publique Hôpitaux de Marseille, Marseille, France

${ }^{11}$ Département d'Anesthésie \& VVC, Gustave Roussy Cancer Center, Villejuif, France

${ }^{12}$ Service d'Anesthésie Réanimation, Groupe Hospitalier Sud, Civils de Lyon, Pierre Benite, France

${ }^{13} \mathrm{CHU}$ Nantes, Université de Nantes, Pôle Anesthésie-Réanimation, Service d'Anesthésie Réanimation Chirurgicale, Hôpital Guillaume et René Laennec, Saint-Herblain, France

${ }^{14} \mathrm{CH}$ Valenciennes, Service d'Anesthésie, Valenciennes, France

${ }^{15} \mathrm{CHU}$ de Nantes, Direction de la Recherche, Plateforme de Méthodologie et Biostatistique, Nantes, France

${ }^{16} \mathrm{CHU}$ Nantes, Service Pharmacie, Hôtel Dieu, Nantes, France

${ }^{17}$ Université de Nantes, Université de Tours, INSERM, SPHERE U1246, Nantes, France

We thank the families and participants who took part in the study as well as the clinical and research staff at the trial sites.

PACMAN study group: Mathieu Oudot, hospital practitioner, Service d’Anesthésie, Centre Hospitalier Départemental Vendée, La Roche Sur Yon, France; Thomas Rimmelé, professor in anaesthesia and critical care medicine, Hôpital Edouard Herriot, Hospice Civils de Lyon Lyon, France; Serge Molliex, professor in anaesthesia and critical care medicine, CHU de Saint Etienne, Service d’Anesthésie-Réanimation, Saint Etienne, France; Olivier Huet, professor in anaesthesia and critical care medicine, CHU de Brest, Université de Brest, Service d’Anesthésie-Réanimation, Brest, France; Vincent Minville, professor in anaesthesia and critical care medicine, CHU Toulouse, Hôpital Purpan, Service d’Anesthésie-Réanimation, Toulouse, France; Bertrand
Dureil, professor in anaesthesia and critical care medicine, $\mathrm{CHU}$ Rouen, Service d’Anesthésie-Réanimation, Rouen, France; Florian Capron, private hospital practitioner, Clinique Jules Verne, Service d'anesthésie, Nantes, France; Benoit Plaud, professor in anaesthesia and critical care medicine, Assistance Publique Hôpitaux de Paris, Hôpital Saint Louis, Paris, France; Sigismond Lasocki, professor in anaesthesia and critical care medicine, CHU d'Angers, Service d’Anesthésie-Réanimation, Angers, France; Pascale Le Maguet, hospital practitioner, $\mathrm{CH}$ de Quimper, Service d'Anesthésie, Quimper, France; and Hélène Beloeil, professor in anaesthesia and critical care medicine, CHU Rennes, Service d’Anesthésie-Réanimation, Rennes, France.

Data and safety monitoring boards: Djillali Annane (Hôpital Raymond-Poincaré, AP/HP, France), Elie Azoulay (Hôpital Saint Louis, AP/HP, France), Jérémie Lefevre (Hôpital Saint Antoine, AP/HP, France) Emilie Vierrion (Université de Nantes, France) and Jean Louis Vincent (Hôpital Erasme, Brussels, Belgium).

Contributors: KA obtained funding from the French Ministry of Health to undertake the study. He is the guarantor and accepts full responsibility for the work. KA and AR conceived, designed, and supervised the study, interpreted the data, and wrote the report. MP and FF performed the statistical analyses. MP was responsible for data management and statistical analysis. All of the authors participated in data collection and acquisition, reviewed the manuscript for important intellectual content; and gave administrative, technical, or material support. Additional contributors were Celine Lerebourg and Delphine Flattres Duchaussoy (research technicians), June Fortin, and Anne Omnes (administrative support, Direction de la recherche clinique Nantes). The lead author had full access to all the data in the study and takes responsibility for the integrity, transparency of the data, and the accuracy of the data analysis. The corresponding author attests that all listed authors meet authorship criteria and that no others meeting the criteria have been omitted.

Funding: This study was funded by the French Ministry of Health (PHRCN 2016, RC16_0442). Nantes University Hospital sponsored the study. The funder of the study had no role in the study design, data collection, data analysis, data interpretation, or writing of the report. There was no agreement concerning confidentiality of the data between the sponsor and the authors. Mylan (which supplied dexamethasone) did not have any role in the conduct of the trial or participate in the analysis, interpretation, or writing and review of the manuscript.

Competing interests: All authors have completed the ICMJE uniform disclosure form at www.icmje.org/coi_disclosure.pdf and declare: support from the French Ministry of Health and Nantes University Hospital for the submitted work; KA reports receiving lecture fees from Baxter, Fisher and Paykel, and LFB and consulting fees from Edward Lifesciences, LFB (Laboratoire français du fractionnement et des biotechnologies). MB reports receiving consulting fees from Becton Dickinson. SI reports receiving personal fees from Drager, Medtronic, Baxter, Medtronic, Fisher \& Paykel, and Fresenius-Xenios. $\mathrm{ML}$ reports receiving consulting fees from Aspen, Orion, MSD, Pfizer, $3 \mathrm{M}$, Octapharma, and Edwards Lifesciences. EF reports receiving consulting fees from Drager Medical, GE Healthcare, Edwards Lifesciences, and Orion Pharma, and lectures fees from Fresenius Kabi, Baxter, and Fisher \& Paykel. AR reports receiving consulting fees from Merck and bioMerieux.

Ethical approval: This trial was approved by Sud Méditerranée V institutional review board in June 2017. The trial was conducted in accordance with the declaration of Helsinki.

Data sharing: Deidentified data about the individual participants will be shared with researchers of further studies. Request for data sharing will be handled in line with the data access and sharing policy of Nantes University Hospital.

The lead author (KA) affirms that the manuscript is an honest, accurate, and transparent account of the study being reported; that no important aspects of the study have been omitted; and that any discrepancies from the study as planned (and, if relevant, registered) have been explained.

Dissemination to participants and related patient and public communities: Participating institutions and anaesthesiology and surgical departments were informed of the results. Results also can be communicated to study participants who express an interest during clinic visits. The results were presented at the French Society of Anaesthesiology and Critical Care Medicine (http://www.sfarlecongres.com/images/Programme-SFAR-2020.pdf). The main results of the current research will be disseminated to related patients and the public through blogs, press releases, newspapers, and broadcasts. 
Provenance and peer review: Not commissioned; externally peer reviewed.

This is an Open Access article distributed in accordance with the Creative Commons Attribution Non Commercial (CC BY-NC 4.0) license, which permits others to distribute, remix, adapt, build upon this work non-commercially, and license their derivative works on different terms, provided the original work is properly cited and the use is noncommercial. See: http://creativecommons.org/licenses/by-nc/4.0/.

1 Weiser TG, Haynes AB, Molina G, et al. Estimate of the global volume of surgery in 2012: an assessment supporting improved health outcomes. Lancet 2015;385(Suppl 2):S11. doi:10.1016/S01406736(15)60806-6

2 Bainbridge D, Martin J, Arango M, Cheng D, Evidence-based Peri-operative Clinical Outcomes Research (EPiCOR) Group. Perioperative and anaesthetic-related mortality in developed and developing countries: a systematic review and metaanalysis. Lancet 2012;380:1075-81. doi:10.1016/S01406736(12)60990-8

3 Peden CJ, Stephens T, Martin G, et al, Enhanced Peri-Operative Care for High-risk patients (EPOCH) trial group. Effectiveness of a national quality improvement programme to improve survival after emergency abdominal surgery (EPOCH): a stepped-wedge clusterrandomised trial. Lancet 2019;393:2213-21. doi:10.1016/S01406736(18)32521-2

4 Pearse RM, Harrison DA, MacDonald N, et al, OPTIMISE Study Group. Effect of a perioperative, cardiac output-guided hemodynamic therapy algorithm on outcomes following major gastrointestinal surgery: a randomized clinical trial and systematic review. JAMA 2014;311:2181-90. doi:10.1001/jama.2014.5305

5 Pearse RM, Moreno RP, Bauer P, et al, European Surgical Outcomes Study (EuSOS) group for the Trials groups of the European Society of Intensive Care Medicine and the European Society of Anaesthesiology. Mortality after surgery in Europe: a 7 day cohort study. Lancet 2012;380:1059-65. doi:10.1016/S01406736(12)61148-9

6 van Berge Henegouwen MI, van der Poll T, van Deventer SJ, Gouma DJ. Peritoneal cytokine release after elective gastrointestinal surgery and postoperative complications. Am / Surg 1998;175:311-6. doi:10.1016/S0002-9610(98)00010-5

7 Lin E, Calvano SE, Lowry SF. Inflammatory cytokines and cell response in surgery. Surgery 2000;127:117-26. doi:10.1067/ msy.2000.101584

8 Annane D, Renault A, Brun-Buisson C, et al, CRICS-TRIGGERSEP Network. Hydrocortisone plus Fludrocortisone for Adults with Septic Shock. N Engl J Med 2018;378:809-18. doi:10.1056/ NEJMoa1705716

9 Roquilly A, Mahe PJ, Seguin P, et al. Hydrocortisone therapy for patients with multiple trauma: the randomized controlled HYPOLYTE study. JAMA 2011;305:1201-9. doi:10.1001/jama.2011.360

10 Asehnoune K, Seguin P, Allary J, et al, Corti-TC Study Group. Hydrocortisone and fludrocortisone for prevention of hospitalacquired pneumonia in patients with severe traumatic brain injury (Corti-TC): a double-blind, multicentre phase 3, randomised placebocontrolled trial. Lancet Respir Med 2014;2:706-16. doi:10.1016/ S2213-2600(14)70144-4

11 Whitlock RP, Devereaux PJ, Teoh KH, et al, SIRS Investigators. Methylprednisolone in patients undergoing cardiopulmonary bypass (SIRS): a randomised, double-blind, placebo-controlled trial. Lancet 2015;386:1243-53. doi:10.1016/S0140-6736(15) 00273-1

12 Yared J-P, Bakri MH, Erzurum SC, et al. Effect of dexamethasone on atrial fibrillation after cardiac surgery: prospective, randomized, double-blind, placebo-controlled trial. / Cardiothorac Vasc Anesth 2007;21:68-75. doi:10.1053/j.jvca.2005.10.014

13 Dieleman JM, Nierich AP, Rosseel PM, et al, Dexamethasone for Cardiac Surgery (DECS) Study Group. Intraoperative high-dose dexamethasone for cardiac surgery: a randomized controlled trial. JAMA 2012;308:1761-7. doi:10.1001/jama.2012.14144

14 DREAMS Trial Collaborators and West Midlands Research Collaborative. Dexamethasone versus standard treatment for postoperative nausea and vomiting in gastrointestinal surgery: randomised controlled trial (DREAMS Trial). BMJ 2017;357:j1455.

15 Polderman JA, Farhang-Razi V, Van Dieren S, et al. Adverse side effects of dexamethasone in surgical patients. Cochrane Database Syst Rev 2018;8:CD011940.

16 Toner AJ, Ganeshanathan V, Chan MT, Ho KM, Corcoran TB. Safety of Perioperative Glucocorticoids in Elective Noncardiac Surgery: A Systematic Review and Metaanalysis. Anesthesiology 2017;126:234-48. doi:10.1097/ ALN.0000000000001466

17 Asehnoune K, Futier E, Feuillet F, Roquilly A, PACMAN group. PACMAN trial protocol, Perioperative Administration of Corticotherapy on Morbidity and mortality After Non-cardiac major surgery: a randomised, multicentre, double-blind, superiority study. BMJ Open 2019;9:e021262. doi:10.1136/bmjopen-2017-021262

18 Smetana GW, Lawrence VA, Cornell JE, American College of Physicians. Preoperative pulmonary risk stratification for noncardiothoracic surgery: systematic review for the American College of Physicians. Ann Intern Med 2006;144:581-95. doi:10.7326/0003-4819-144-8-200604180-00009

19 Weber WP, Mujagic E, Zwahlen M, et al. Timing of surgical antimicrobial prophylaxis: a phase 3 randomised controlled trial. Lancet Infect Dis 2017;17:605-14. doi:10.1016/S14733099(17)30176-7

20 Futier E, Constantin J-M, Paugam-Burtz C, et al, IMPROVE Study Group. A trial of intraoperative low-tidal-volume ventilation in abdominal surgery. N Engl J Med 2013;369:428-37. doi:10.1056/ NEJMoa1301082

21 Futier E, Lefrant J-Y, Guinot P-G, et al, INPRESS Study Group. Effect of Individualized vs Standard Blood Pressure Management Strategies on Postoperative Organ Dysfunction Among High-Risk Patients Undergoing Major Surgery: A Randomized Clinical Trial. JAMA 2017;318:1346-57. doi:10.1001/jama.2017.14172

22 Chanques G, Conseil M, Roger C, et al, SOS-Ventilation study investigators. Immediate interruption of sedation compared with usual sedation care in critically ill postoperative patients (SOS Ventilation): a randomised, parallel-group clinical trial. Lancet Respir Med 2017:5:795-805 doi:10.1016/S2213-2600(17)30304-1

23 Singer M, Deutschman CS, Seymour CW, et al. The Third International Consensus Definitions for Sepsis and Septic Shock (Sepsis-3). IAMA 2016:315:801-10. doi:10.1001/jama.2016.0287

24 Jaber S, Lescot T, Futier E, et al, NIVAS Study Group. Effect of Noninvasive Ventilation on Tracheal Reintubation Among Patients With Hypoxemic Respiratory Failure Following Abdominal Surgery: A Randomized Clinical Trial. JAMA 2016;315:1345-53. doi:10.1001/ jama.2016.2706

25 Polderman JAW, Farhang-Razi V, van Dieren S, et al. Adverse sideeffects of dexamethasone in surgical patients - an abridged Cochrane systematic review. Anaesthesia 2019;74:929-39. doi:10.1111/ anae.14610

26 Lepelletier Y, Zollinger R, Ghirelli C, et al. Toll-like receptor contro of glucocorticoid-induced apoptosis in human plasmacytoid predendritic cells (pDCs). Blood 2010;116:3389-97. doi:10.1182/ blood-2010-05-282913

27 Bouras M, Asehnoune K, Roquilly A. Contribution of Dendritic Cell Responses to Sepsis-Induced Immunosuppression and to Susceptibility to Secondary Pneumonia. Front Immunol 2018:9.2590 doi:10.3389/fimmu 2018.02590

28 Keh D, Boehnke T, Weber-Cartens S, et al. Immunologic and hemodynamic effects of "low-dose" hydrocortisone in septic shock: a double-blind, randomized, placebo-controlled, crossover study. Am J Respir Crit Care Med 2003;167:512-20. doi:10.1164/ rccm.200205-4460C

29 Roquilly A, Broquet A, Jacqueline C, et al. Hydrocortisone prevents immunosuppression by interleukin-10+ natural killer cells after trauma-hemorrhage. Crit Care Med 2014;42:e752-61. doi:10.1097/ CCM.0000000000000658

30 Kaufmann I, Briegel J, Schliephake F, et al. Stress doses of hydrocortisone in septic shock: beneficial effects on opsonizationdependent neutrophil functions. Intensive Care Med 2008;34:344-9. doi:10.1007/s00134-007-0868-8

31 Annane D, Bellissant E, Bollaert PE, et al. Corticosteroids for treating sepsis in children and adults. Cochrane Database Syst Rev 2019;12:CD002243. doi:10.1002/14651858.CD002243.pub4

32 Meijvis SC, Hardeman H, Remmelts HH, et al. Dexamethasone and length of hospital stay in patients with community-acquired pneumonia: a randomised, double-blind, placebo-controlled trial. Lancet 2011;377:2023-30. doi:10.1016/S0140-6736(11) 60607-7

33 Horby P, Lim WS, Emberson JR, et al, RECOVERY Collaborative Group. Dexamethasone in Hospitalized Patients with Covid-19. N Engl J Med 2021:384:693-704. doi:10.1056/NEJMoa2021436

34 Srinivasa S, Kahokehr AA, Yu T-C, Hill AG. Preoperative glucocorticoid use in major abdominal surgery: systematic review and meta-analysis of randomized trials. Ann Surg 2011:254:183-91. doi:10.1097/ SLA.0b013e3182261118

35 Weijs TJ, Dieleman JM, Ruurda JP, Kroese AC, Knape HJTA, van Hillegersberg R. The effect of perioperative administration of glucocorticoids on pulmonary complications after transthoracic oesophagectomy: a systematic review and metaanalysis. Eur / Anaesthesiol 2014;31:685-94. doi:10.1097/ ElA.0000000000000093

36 Corcoran TB, Edwards T. A survey of antiemetic dexamethasone administration-frequency of use and perceptions of benefits and risks. Anaesth Intensive Care 2015;43:167-74. doi:10.1177/0310057X1504300205 
37 Gandhi GY, Nuttall GA, Abel MD, et al. Intraoperative hyperglycemia and perioperative outcomes in cardiac surgery patients. Mayo Clin Proc 2005;80:862-6. doi:10.4065/80.7.862

38 Coursin DB, Connery LE, Ketzler JT. Perioperative diabetic and hyperglycemic management issues. Crit Care Med 2004;32(Suppl):S116-25. doi:10.1097/01. CCM.0000115623.52021.CO

39 Frisch A, Chandra P, Smiley D, et al. Prevalence and clinical outcome of hyperglycemia in the perioperative period in noncardiac surgery. Diabetes Care 2010;33:1783-8. doi:10.2337/dc10-0304

40 Lomivorotov V, Kornilov I, Boboshko V, et al. Effect of Intraoperative Dexamethasone on Major Complications and Mortality Among Infants
Undergoing Cardiac Surgery: The DECISION Randomized Clinical Trial. JAMA 2020;323:2485-92. doi:10.1001/jama.2020.8133

41 Pastores SM, Annane D, Rochwerg B, Corticosteroid Guideline Task Force of SCCM and ESICM. Guidelines for the diagnosis and management of critical illness-related corticosteroid insufficiency (CIRCI) in critically ill patients (Part II): Society of Critical Care Medicine (SCCM) and European Society of Intensive Care Medicine (ESICM) 2017. Intensive Care Med 2018;44:474-7. doi:10.1007/ s00134-017-4951-5

Web appendix: Supplementary materials 